\title{
Molecular Imprinting Based Hybrid Ratiometric Fluorescence Sensor for the Visual Determination of Bovine Hemoglobin
}

\author{
Xiaoyan Wang, ${ }^{*}{ }^{\dagger},+$ Shuangmei Yu, ${ }^{\S}$ Wen Liu, ${ }^{\ddagger}$ Longwen $\mathrm{Fu}^{\ddagger}{ }^{\ddagger}$ Yunqing Wang, ${ }^{\ddagger}$ Jinhua Li, \\ and Lingxin Chen*, \\ ${ }^{\dagger}$ School of Pharmacy, Binzhou Medical University, Yantai 264003, China \\ ${ }^{\ddagger}$ Key Laboratory of Coastal Environmental Processes and Ecological Remediation, Yantai Institute of Coastal Zone Research, Chinese \\ Academy of Sciences, Yantai 264003, China \\ ${ }^{\S}$ Radiotherapy Ward, Yantai Yuhuangding Hospital, Yantai 264000, China
}

\section{Supporting Information}

\begin{abstract}
We describe a simple and effective strategy to construct a molecular imprinting ratiometric fluorescence sensor (MIR sensor) for the visual detection of bovine hemoglobin $(\mathrm{BHb})$ used as a model protein. The sensor was prepared by simply mixing the solution of green and red CdTe quantum dots (QDs), which were embedded in core-shell structured molecularly imprinted polymers and silica nanoparticles, respectively. The resultant hybrid MIR sensor can selectively bind with $\mathrm{BHb}$ and thus quench the fluorescence of the green QDs, while the red QDs wrapped with silica are insensitive to $\mathrm{BHb}$ with the fluorescence intensity unchanged. As a result, a continuous obvious fluorescence color change from green to red can be observed by naked eyes, with the

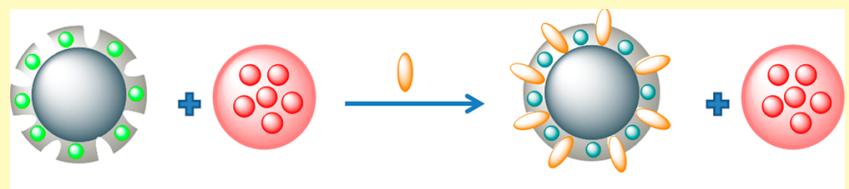
detection limit of $9.6 \mathrm{nM}$. Moreover, the MIR sensor was successfully applied to determine BHb in bovine urine samples with satisfactory recoveries at three spiking levels ranging from 95.7 to $101.5 \%$, indicating great potential application for detecting $\mathrm{BHb}$ in real samples. This strategy of using different fluorescence emission materials incorporated to construct a ratiometric fluorescence sensor is reasonable and convenient, which can be extended to the preparation of other ratiometric fluorescence systems for targeted analytes.
\end{abstract}

KEYWORDS: molecular imprinting, protein imprinting, ratiometric fluorescence, bovine hemoglobin, quantum dots

$\mathrm{H}$ emoglobin $(\mathrm{Hb})$ plays an important role in a variety of physiological activities. It is a conjugated iron-protein compound in red blood cells and participates in the transportation of oxygen, carbon dioxide, and nitric oxide. ${ }^{1} \mathrm{~A}$ deficiency of hemoglobin can result in various diseases, such as anemia, thalassemia, and so on. ${ }^{2}$ Thus, to analyze $\mathrm{Hb}$ is of important significance for clinical diagnoses, and biomedical and proteomics research. Because the similarity between bovine hemoglobin $(\mathrm{BHb})$ and $\mathrm{Hb}$ is as high as $90 \%,{ }^{2,3} \mathrm{BHb}$ can be used as the target protein instead of $\mathrm{Hb}$ in the analysis of $\mathrm{Hb}$. At present, protein detection from complex matrices is still a challenging issue. So, it is essential to ameliorate the detection selectivity toward target proteins.

Molecular imprinting technology is famous for its ability in providing and improving selectivity, which has been demonstrated by the creation of molecularly imprinting polymers (MIPs) with tailor-made binding sites complementary to the template molecules in shape, size, and functional groups. ${ }^{4}$ Owing to high selectivity, good physical stability, easy preparation, and low cost, MIPs have gained popularity in many fields, including sample pretreatment/chromatographic separation and chemical/biological sensing. ${ }^{4}$ So far, the imprinting of small molecules has attained great success; however, the preparation of MIPs for biomacromolecules, like proteins, still remains challengeable due to the large molecular size, complexity, flexible conformation, and solubility. ${ }^{5,6}$ To solve the above problems, various strategies have been developed, such as surface imprinting, ${ }^{7,8}$ boronate affinity molecular imprinting, ${ }^{9,10}$ and epitope imprinting. ${ }^{11,12}$ Among them, surface imprinting is promising, because the imprinting cavities on or near the support surfaces can facilitate removal and adsorption of the template. Moreover, when combined with support nanomaterials, e.g., $\mathrm{SiO}_{2}$ and quantum dots (QDs), the obtained MIPs not only get enhanced density of surface binding sites, but also have regular morphologies in favor of chemical modifications, rendering MIPs tailored for sensors.

Received: October 30, 2017

Accepted: January 16, 2018

Published: January 16, 2018 
Scheme 1. Schematic Illustration of the Proposed Synthetic and Construction Strategy for MIPs Ratiometic Sensor

A
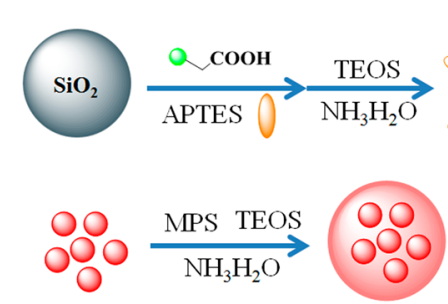
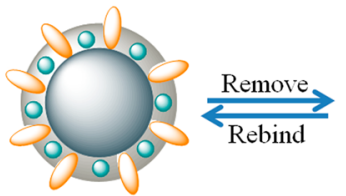

$\mathrm{CdTe} O \mathrm{SiO}_{2}$

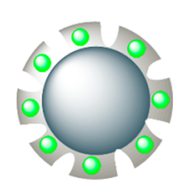

QDs 0 BHb O QDs

CdTe MIPs

B

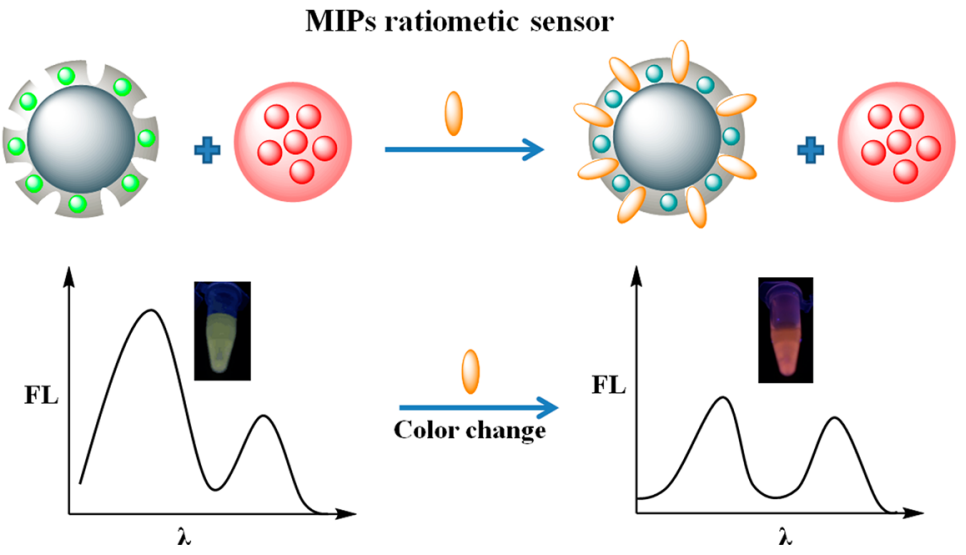

Development of MIPs based fluorescence sensors is of great concern by virtue of the combination high selectivity of MIPs with high sensitivity of fluorescence detection. Among fluorescent label materials, semiconductor QDs enjoy great popularity, thanks to their bright photoluminescence, narrow and symmetric emission with tunable colors, and high photostability. ${ }^{13-15}$ Some groups have reported QDs@MIPs fluorescence sensors for proteins, such as bovine hemoglobin, ${ }^{16-18}$ lysozyme, ${ }^{19,20}$ cytochrome $c{ }^{12,21}$ and $\alpha$-fetoprotein. $^{22,23}$ Compared to conventional sole responsive fluorescence detection, ratiometric fluorescence technique using dual fluorescence signals has recently attracted increasing attention, since it can eliminate environmental effects and give improved sensitivity for trace level analytes. ${ }^{24-26}$ Excitingly, novel MIPs based ratiometric fluorescence sensors have been reported and displayed the potential in practical application. For example, Xu's and Chen's groups have respectively carried out related research on MIPs ratiometric fluorescence sensors for TNT, ${ }^{27}$ melamine, ${ }^{28}$ and bisphenol $\mathrm{A}^{29}$ and for phycocyanin ${ }^{30}$ and 2,4dichlorophenoxyacetic acid. ${ }^{31}$ These studies have confirmed that MIPs ratiometric fluorescence sensors can obtain high sensitivity and visual recognition by the naked eye.

In general, building ratiometric fluorescence sensors needs two individual materials with different fluorescence emission wavelengths. A simple approach is to use one emission peak as response signal to the target analyte and the other as reference signal, leading to the color change of the two emission fluorescent materials obviously and easily observed by the naked eye. Since the ratiometric fluorescence response relies on the fluorescence intensity of the two individual fluorescence materials, which is related to their amounts, it is imperative to optimize their proportions. However, optimization during the preparation process of core-shell MIPs ratiometric fluorescence sensors is usually hard to control for ideal color change, relatively time-consuming, and laborious. ${ }^{27,28,31}$
Herein, we developed a simple and easy way to construct MIPs ratiometric fluorescence sensor (MIR sensor) for the visual detection of $\mathrm{BHb}$, through simply mixing two solutions of green and red CdTe QDs, which were embedded in coreshell structured MIPs (CdTe@MIPs) and silica nanoparticles ( $\left.\mathrm{CdTe} @ \mathrm{SiO}_{2}\right)$, respectively. $\mathrm{BHb}$ as imprinting model protein demonstrated the proof-of-principle of our strategy. Two different sizes of CdTe QDs emitting green and red fluorescence were used as response signal and reference signal, respectively. Green CdTe@MIPs and red $\mathrm{CdTe} @ \mathrm{SiO}_{2}$ were synthesized separately, and then they were mixed according to an appropriate proportion for obtaining the ratiometric fluorescent system. Upon the addition of BHb, CdTe@MIPs could bind $\mathrm{BHb}$, resulting in the fluorescence intensity decreasing gradually with the increase of $\mathrm{BHb}$ concentration. While the fluorescence of $\mathrm{CdTe} @ \mathrm{SiO}_{2}$ slightly fluctuated in the presence of $\mathrm{BHb}$, which was negligible when compared with that of CdTe@MIPs. The variation of fluorescence intensity ratios resulted in a continuous fluorescence color change from green to red, allowing the visual detection of $\mathrm{BHb}$. Such mixedtype construction effectively avoided time-consuming synthesis of composite nanoparticles, providing a simple, convenient and alternative construction approach to ratiometric fluorescence sensors.

\section{EXPERIMENTAL SECTION}

Reagents and Materials. Tellurium powder, cadmium nitrate $\left(\mathrm{Cd}\left(\mathrm{NO}_{3}\right)_{2}\right)$, sodium hydroxide, ethanol, ammoniumhydroxide $(25 \%)$, acetonitrile (ACN), tetraethoxysilicane (TEOS) 3-aminopropyltriethoxysilane (APTES), 3-mercapto-propyltrimethoxysilane (MPS), and phosphate buffered saline (PBS) were supplied by Sinopharm Chemical Reagent Co. Ltd. (Shanghai, China). Sodium borohydride $\left(\mathrm{NaBH}_{4}\right)$, bovine hemoglobin $(\mathrm{BHb})$, bovine serum albumin (BSA), lysozyme (Lyz), protamine, and thioglycollic acid (TGA) were purchased from Sigma-Aldrich (Shanghai, China). 
Instrumentation. Fluorescence spectra were recorded by using a Fluoromax-4 spectrofluorometer (Horiba Scientific). The morphological evaluation was taken on a transmission electron microscope (TEM, JEM-1230, operating at $100 \mathrm{kV}$ ) and scanning electron microscope (SEM, Hitachi S-4800 FE-SEM, operating at $5 \mathrm{kV}$. FT-IR analyses were recorded by using a FT-IR spectrometer (Thermo Nicolet Corporation, USA). Zeta potential and dynamic light scattering (DLS) tests were performed on a Malvern Zetasizer Nano-ZS90 (ZEN3590, UK). Elemental analysis was measured using a Vario Microcube element analyzer (Elementar Company, Germany).

Preparation of Red CdTe QDs Embedded Silica Nanoparticles $\left(\mathrm{CdTe} @ \mathrm{SiO}_{2}\right)$. TGA-modified red CdTe QDs were obtained according to our previous synthesis procedure for QDs, ${ }^{15}$ and changing the refluxing time from 2 to $48 \mathrm{~h}$. Then, $\mathrm{CdTe} @ \mathrm{SiO}_{2}$ was synthesized by sol-gel process according to the reported method with some modifications ${ }^{26}$ as below. Typically, $40 \mathrm{~mL}$ of ethanol and 5 $\mathrm{mL}$ of red CdTe QDs solution were mixed and stirred for $10 \mathrm{~min}$. Then $20 \mu \mathrm{L}$ of MPS was added into the above solution. After stirring for $12 \mathrm{~h}, 200 \mu \mathrm{L}$ of $\mathrm{NH}_{3} \cdot \mathrm{H}_{2} \mathrm{O}$ and $200 \mu \mathrm{L}$ of TEOS were added and stirring continued for $5 \mathrm{~h}$. Finally, the products were centrifuged and washed with ethanol and ultrapure water.

Preparation of BHb Imprinted Nanoparticles (CdTe@MIPs). TGA-modified green CdTe QDs were synthesized according to our previous procedure, ${ }^{15}$ and $\mathrm{SiO}_{2}$ nanoparticles were synthesized according to our report. ${ }^{30}$ Then, $2 \mathrm{~mL}$ of the green CdTe QDs was added to $18 \mathrm{~mL}$ of ultrapure water containing $800 \mu \mathrm{L}$ of $12.5 \mathrm{mg} / \mathrm{mL}$ $\mathrm{SiO}_{2}$. After stirring for $30 \mathrm{~min}, 10 \mathrm{mg}$ of $\mathrm{BHb}$ and $20 \mu \mathrm{L}$ APTES were added into the above solution; after stirring for $1 \mathrm{~h}, 40 \mu \mathrm{L}$ of $\mathrm{NH}_{3}$. $\mathrm{H}_{2} \mathrm{O}$ and $40 \mu \mathrm{L}$ of TEOS were added and stirring continued overnight in the dark. Finally, the resultant CdTe@MIPs were centrifuged and washed with ethanol/acetonitrile $(8: 2, \mathrm{v} / \mathrm{v})$ to remove $\mathrm{BHb}$, and then washed with ultrapure water three times. As a control, its corresponding nonimprinted polymers (CdTe@NIPs) were synthesized using the same procedure without the template $\mathrm{BHb}$.

Construction of the Molecular Imprinting Ratiometric Fluorescence Sensor (MIR Sensor). To obtain the MIR sensor for BHb determination, $100 \mu \mathrm{L}$ of CdTe@MIPs solution $(2 \mathrm{mg} / \mathrm{mL})$ and $20 \mu \mathrm{L}$ of the $\mathrm{CdTe} @ \mathrm{SiO}_{2}$ solution were mixed thoroughly in 1 $\mathrm{mL}$ of PBS solution. The fluorescence intensity of the MIR sensor was evaluated under a single wavelength excitation at $420 \mathrm{~nm}$ with a recording emission range of $440-700 \mathrm{~nm}$.

Analysis of Real Samples. Urine samples were randomly collected from a healthy bovine from a rural household (Local in Yantai City, China). The real urine samples were filtered with a 0.45 $\mu \mathrm{m}$ microfiltration membrane to remove any possible particulate suspension, and then they were diluted 100 -fold for spiking. Recovery experiments were performed by using the spiked $\mathrm{BHb}$ at three concentration levels of $0.3,1.0$, and $2.0 \mu \mathrm{mol} \mathrm{L}^{-1}$, and then these samples were analyzed using the MIR sensor. All the tests were performed in triplicate.

\section{RESULTS AND DISCUSSION}

Construction and Characterization of Ratiometric Fluorescent Sensor. The synthesis of CdTe@MIPs, $\mathrm{CdTe} @ \mathrm{SiO}_{2}$ and the construction of the dual emission fluorescence nanohybrid for visual detection of $\mathrm{BHb}$ were illustrated in Scheme 1; CdTe@MIPs and CdTe@SiO $\mathrm{S}_{2}$ were synthesized separately (Scheme 1A) and then mixed in an appropriate proportion (Scheme 1B). By using silica nanoparticles as support for surface imprinting, $\mathrm{BHb}$ as imprinted template, APTES as functional monomer, TGA-modified green CdTe QDs as assistant functional monomer, and TEOS as cross-linker, the core-shell structured CdTe@MIPs were prepared. The as-prepared CdTe@MIPs exhibited an emission maximum at $541 \mathrm{~nm}$ with a strong green fluorescence (Figure 1a). CdTe@SiO ${ }_{2}$ prepared by sol-gel process displayed an emission maximum at $647 \mathrm{~nm}$ with a strong red fluorescence (Figure 1b). When CdTe@MIPs were mixed with CdTe@ $@ \mathrm{SiO}_{2}$

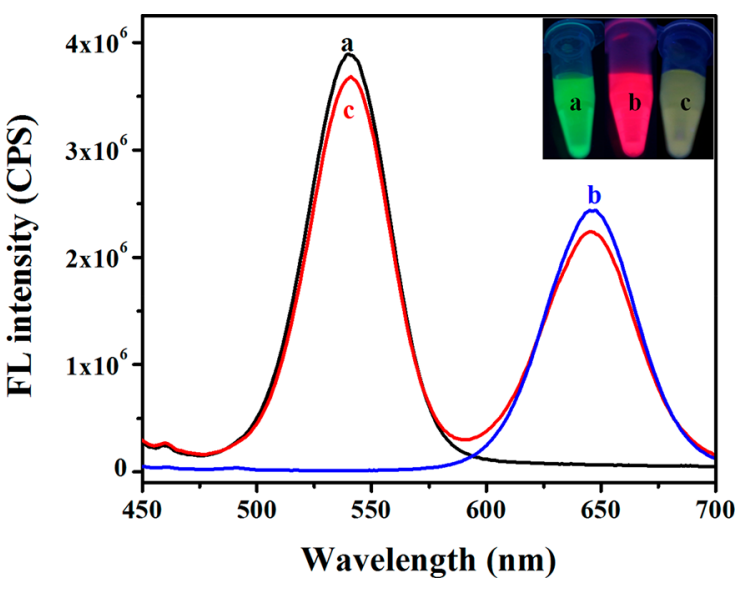

Figure 1. Fluorescence emission spectra $\left(\lambda_{\text {ex }}=420 \mathrm{~nm}\right)$ of (a) CdTe@MIPs, (b) CdTe@SiO ${ }_{2}$, and (c) MIPs ratiometic sensor, respectively. The inset shows the corresponding fluorescence images under a $365 \mathrm{~nm}$ UV lamp.

in a certain proportion, producing an appropriate fluorescence color ratio, a new MIR sensor was obtained, which displayed dual fluorescence emission peaks at 541 and $647 \mathrm{~nm}$ under a single excitation at $420 \mathrm{~nm}$ (Figure 1c). Since there were recognition sites on the surface of CdTe@MIPs, BHb could enter the recognition sites, quenching the fluorescence of the green QDs. The fluorescence emission spectrum of CdTe@ MIPs had no spectral overlap with the absorption spectrum of $\mathrm{BHb}$ (Figure S1), so the fluorescence quenching of QDs could be ascribed to the electron transfer between $\mathrm{QDs}$ and $\mathrm{BHb}$. With the presence and increase of $\mathrm{BHb}$, the green fluorescence of QDs was quenched gradually. However, the red QDs wrapped with silica would prevent the direct contact with the external $\mathrm{BHb}$. There are no carboxyl and amino groups on the surface of $\mathrm{CdTe} @ \mathrm{SiO}_{2}$, so red fluorescence of QDs was insensitive to $\mathrm{BHb}$. As a result, a variation of fluorescence intensity ratios was shown and a distinct fluorescence color change from green to red could be used for the ratiometric detection of $\mathrm{BHb}$.

The morphological structures of $\mathrm{SiO}_{2}, \mathrm{CdTe} @ \mathrm{MIPs}, \mathrm{CdTe} @$ NIPs and CdTe@SiO $\mathrm{O}_{2}$ were investigated by TEM and SEM. As shown in Figure 2, $\mathrm{SiO}_{2}$ nanoparticles had good dispersion with an average diameter of 50-60 $\mathrm{nm}$ and their surface was very smooth (Figure 2a, d). The CdTe@MIPs and CdTe@NIPs had similar morphologies and displayed rough polymeric surface (Figure 2b, c, e, and f). The MIPs layer could be estimated to $<10 \mathrm{~nm}$ in thickness calculated from the TEM image. Besides, CdTe@SiO ${ }_{2}$ also had good dispersion with an average diameter of $30-40 \mathrm{~nm}$. The size distributions of $\mathrm{SiO}_{2}$, CdTe@MIPs, and CdTe@NIPs were obtained by DLS measurement as shown in Figure S2. As seen, the intensity contribution versus diameters of the above nanoparticles showed good size-distribution and the main distribution peak of $\mathrm{SiO}_{2}$ was about $54 \mathrm{~nm}$, and the dominant distribution peak of CdTe@MIPs and CdTe@NIPs was around $60 \mathrm{~nm}$, which were consistent with TEM results. These indicated that they could well disperse in aqueous solution.

Furthermore, elemental analysis was performed to examine the nitrogen, carbon, hydrogen and sulfur content (Table S1). As seen, there was no sulfur element in $\mathrm{SiO}_{2}$; however, it existed in CdTe@MIPs and CdTe@NIPs, which indicated that TGA-QDs were successfully introduced. The marked enlarge- 

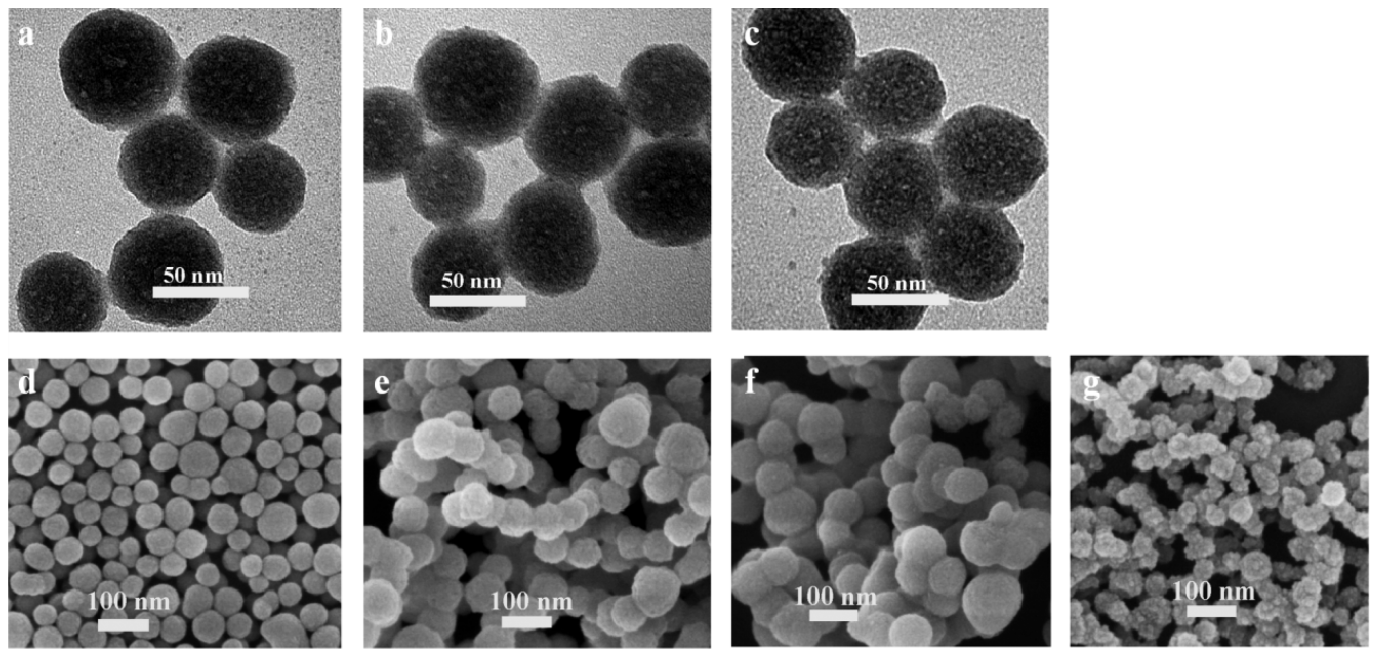

Figure 2. TEM and SEM images of the synthesized (a, d) $\mathrm{SiO}_{2}$, (b, e) CdTe@MIPs, (c, f) CdTe@NIP, and (g) CdTe@SiO .

ment in the nitrogen atomic composition from $0.411 \%$ for $\mathrm{SiO}_{2}$ to $2.7785 \%, 2.486 \%$ for CdTe@MIPs and CdTe@NIPs, respectively, which revealed that amine groups were successfully introduced onto the surface of $\mathrm{SiO}_{2}$. Besides, the carbon and hydrogen atomic compositions for CdTe@MIPs and CdTe@NIPs also increased significantly compared with $\mathrm{SiO}_{2}$ and the results indicated that the existence of carboxyl groups in the CdTe@MIPs and CdTe@NIPs. Overall, the CdTe@ MIPs and CdTe@NIPs were successfully prepared.

The successful preparation of CdTe@MIPs and CdTe@ NIPs was also proved through the existence of carboxyl groups and amino groups as shown in the FT-IR spectra (Figure S3). As seen, the peak at $1469 \mathrm{~cm}^{-1}$ was the characteristic peak of the propylamino groups. The peaks at 1545 and $1388 \mathrm{~cm}^{-1}$ quite possibly corresponded with the antisymmetrical and symmetric vibrations of $\mathrm{COO}^{-}$, respectively. ${ }^{32}$ All these characteristic bands in the spectra suggested the existence of TGA-QDs and APTES in the CdTe@MIPs and CdTe@NIPs.

The surface charge of the synthesized $\mathrm{SiO}_{2}, \mathrm{CdTe} @ M I P s$ and CdTe@NIPs particles was measured by a zeta potentiometer. The surface charge of $\mathrm{SiO}_{2}$ particles is $-35 \mathrm{mV}$, and the surface charge of CdTe@MIPs and CdTe@NIPs was 27.8 $\mathrm{mV}$ and $32.5 \mathrm{mV}$, respectively. Because the surface of $\mathrm{SiO}_{2}$ is mainly hydroxyl, which makes its surface with a strong negative power. After the modification of $\mathrm{SiO}_{2}$ with amino groups, the shell is strong positive due to amino groups with positive. All above results indicated CdTe@MIPs and CdTe@NIPs was successfully synthesized.

Ratio Optimal for CdTe@MIPs and CdTe@SiO 2 in the MIR Sensor. The ratiometric fluorescence response of the MIR sensor relied on the fluorescence intensity at 541 and 647 $\mathrm{nm}$, which was related to the proportion of CdTe@MIPs and CdTe@SiO 2 , respectively. When the ratio of CdTe@MIP and $\mathrm{CdTe} @ \mathrm{SiO}_{2}$ was 10:1, it could only lead to a fluorescent color change from green to orange upon addition of $\mathrm{BHb}$ (Figure S4A). A wide color change from green to red was obtained for the MIR sensor with a ratio of 5:1 (Figure 4A). Continuous increase of the ratio to 10:3 only resulted in color change from yellow to red (Figure S4B). Thus, the hybrid consisting of 5:1 of CdTe@MIPs and CdTe@SiO 2 was selected to build the MIR sensor for $\mathrm{BHb}$ detection.

Condition Optimization for the MIR Sensor. Fluorescence stability, $\mathrm{pH}$ and response time were three major parameters, which could highly influence the fluorescence properties of the MIR sensor, and optimized as below. The stability of the MIR sensor was tested by fluorescence spectra. The fluorescence intensity ratios $\left(I_{541} / I_{647}\right)$ of the ratiometric sensor remained almost unchanged over $2 \mathrm{~h}$ (Figure S5), displaying its good fluorescence stability. The influence of $\mathrm{pH}$ was examined by $\left(F_{0}-F\right) / F$ in the range of 5.0-9.0, as shown in Figure $S 6$; herein, $F_{0}$ and $F$ stand for the fluorescence intensity of green QDs in the absence and presence of the $\mathrm{BHb}$, respectively. It was seen from the figure that the quenching efficiency was affected at different $\mathrm{pH}$ values. As $\mathrm{pH}$ increased, the quenching efficiency first increased and then decreased. Under $\mathrm{pH}$ 8.0, the quenching efficiency was the maximum, possibly owing to the same condition as CdTe@MIPs synthesis. At the $\mathrm{pH}$ of $8, \mathrm{BHb}$ with negative charges most readily bound with amino groups of APTES, just as Turner et al. reported, ${ }^{33}$ "optimum binding occurs when polymer is exposed to the same conditions as those used for polymerization". Consequently, the most remarkable quenching occurred. Besides, under high or low $\mathrm{pH}, \mathrm{BHb}$ may be denatured. So, the optimum $\mathrm{pH}$ of 8 was used for the following work.

Furthermore, to investigate the kinetics of the MIR sensor, the fluorescence intensity at about $541 \mathrm{~nm}$ was recorded at different reaction time after adding $\mathrm{BHb}$ (Figure $\mathrm{S} 7$ ). With the addition of $\mathrm{BHb}$, the fluorescence intensity at about $541 \mathrm{~nm}$ was decreased rapidly and then kept unchanged in less than 10 min, demonstrating that $\mathrm{BHb}$ could quickly reach the imprinted cavities, which could well attribute to the ultrathin imprinting layer. Hence, $10 \mathrm{~min}$ was chosen as the reaction/incubation time.

Sensitivity and Selectivity of the MIR Sensor. The sensitivity of the MIR sensor was evaluated upon addition of different amounts of $\mathrm{BHb}$. As seen from Figure $3 \mathrm{~A}$, the fluorescence intensity of the green QDs gradually decreased along with increasing the concentration of $\mathrm{BHb}$, while the fluorescence intensity of the red QDs kept almost unchanged. Owing to the red QDs as reference, with the decrease of green QDs intensity, the ratios of the two fluorescence intensities varied, leading to a distinguishable fluorescence color change from light green to orange red and to red, which could be clearly observed by the naked eye. As can be seen in Figure 3a, a good linear relationship was obtained by plotting the ratio of 

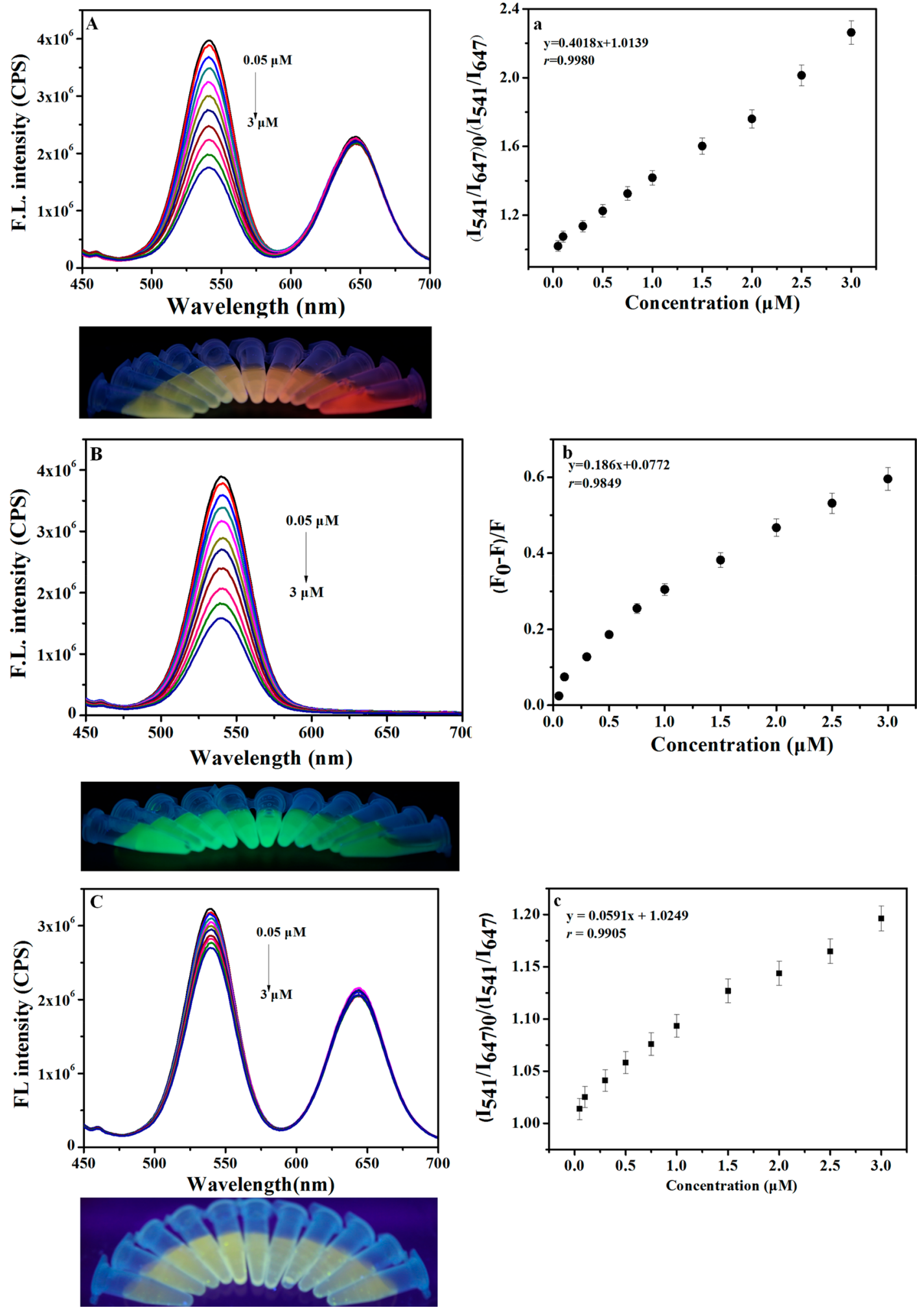

Figure 3. Fluorescence spectra and corresponding fluorescence intensity ratio of (A,a) MIPs ratiometic sensor, (B,b) only CdTe@MIPs, and (C,c) NIPs ratiometic sensor upon the addition of different amounts of BHb; The ratio of CdTe@MIPs or CdTe@NIPs and CdTe@SiO $\mathrm{C}_{2}$ is 5:1. The images in the bottom are their corresponding fluorescence images taken under a UV lamp $(365 \mathrm{~nm})$. The concentrations of BHb from left to right are $0,0.05,0.1,0.3,0.5,0.75,1,1.5,2,2.5$, and $3 \mu \mathrm{M}$, respectively.

the green and red QDs fluorescence intensity $\left(I_{541} / I_{647}\right)_{0} /\left(I_{541} /\right.$ $\left.I_{647}\right)$ versus the concentrations of $\mathrm{BHb}$ within $0.050-3.0 \mu \mathrm{M}$. The limit of detection (LOD) was down to $9.6 \mathrm{nM}$, on the basis of $3 \sigma / s$, in which $\sigma$ means the standard deviation of the blank measurements, and $s$ means the slope of calibration curve. $^{31}$

In order to demonstrate the advantages of the MIR sensor, single-green emission of CdTe@MIPs was determined similarly. As seen in Figure 3B, with the addition and increase 


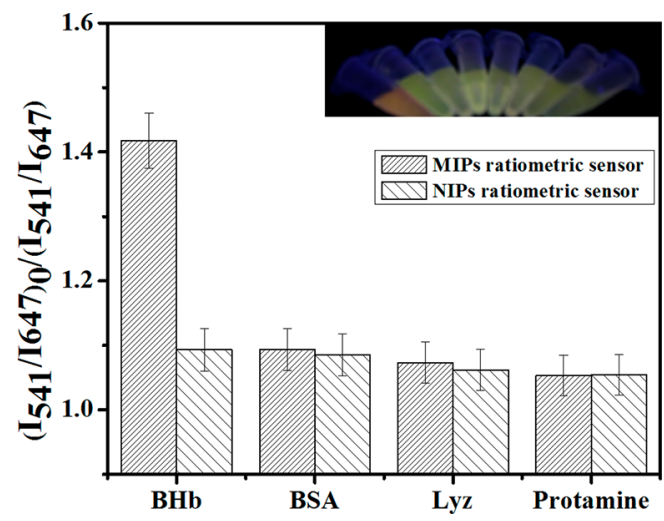

Figure 4. Selectivity of MIPs ratiometic sensor and NIPs ratiometic sensor for $\mathrm{BHb}$ over other analogues including BSA, Lyz, and Protamine at concentration of $1 \mu \mathrm{M}$.

Table 1. Results of Spiked Recoveries and RSDs (\%; $n=3$ ) for Determination of $\mathrm{BHb}$ in Bovine Urine Samples Using the Constructed MIR Sensor

\begin{tabular}{cccc}
\hline sample & added $(\mu \mathrm{M})$ & found $(\mu \mathrm{M})$ & recovery \pm RSD $(\%)$ \\
bovine urine & 0 & 0 & \\
& 0.3 & 0.287 & $95.7 \pm 3.6$ \\
& 1.0 & 0.981 & $98.1 \pm 3.1$ \\
& 2.0 & 2.03 & $101.5 \pm 4.2$
\end{tabular}

of $\mathrm{BHb}$, the fluorescence intensity of green QDs decreased, resulting in the color change of the green QDs fluorescence quenching being hard to distinguish by the naked eye compared with that of the MIR sensor. Moreover, as displayed in Figure 3b, the single fluorescence quenching of CdTe@MIPs sensor was less sensitive than that of the MIR sensor for the visual detection of $\mathrm{BHb}$.

The sensitivity of the NIPs ratiometric sensor was also examined as shown in Figure 3C. The green fluorescence intensity of NIPs ratiometric sensor could also be quenched with the addition of $\mathrm{BHb}$; however, the decrease of fluorescence intensity was not obvious compared with the MIR sensor under the same concentration of $\mathrm{BHb}$, which could be attributed to that there no specific recognition site was in the NIPs. As a result, the decrease of green fluorescence intensity was not large enough to change colors and it was hard to be distinguished by the naked eye. Accordingly, a high imprinting factor was obtained of 6.8 , calculated as the ratio of the slopes of linear relationship of ratiometric MIPs and NIPs.

In the meanwhile, the selectivity of the MIR sensor for $\mathrm{BHb}$ was also assessed by recording the fluorescence intensity ratios $\left(I_{541} / I_{647}\right)$ of the sensor at the same concentration with other proteins (BSA, Lyz, Protamine). As shown from Figure 4, the fluorescence intensity at $541 \mathrm{~nm}$ of the MIR sensor was highly quenched by $\mathrm{BHb}$ and orange red fluorescence color was present. Conversely, the value of $I_{541} / I_{647}$ and the fluorescence color have no obvious change with other proteins presence at 1 $\mu \mathrm{M}$. The reason is mainly because that the MIR sensor has specific recognition sites in shape, size, and functional groups with the template $\mathrm{BHb}$, and can easily bind with imprinted sites, quenching the fluorescence of green QDs. Whereas, other proteins were not complementary to the specific recognition sites and thereby could not effectively influence the fluorescence intensity of green QDs. The results suggested the MIR sensor had good selectivity and specificity for $\mathrm{BHb}$.

Practical Applicability and Method Performance Comparison. The practical applicability of the MIR sensor was further studied in urine sample spiked with different concentration of $\mathrm{BHb}$. It was clearly shown in Table 1 that the recoveries ranged from $95.7-101.5 \%$ with relative standard deviations (RSDs) of $3.1-4.2 \%$ by the MIR sensor. So, the MIR sensor was demonstrated practically applicable and reliable for sensitive and accurate determination of low $\mathrm{BHb}$ contents in real samples.

The strategy and performance of our developed MIR sensor for detection of $\mathrm{BHb}$ was compared with other different MIPsbased fluorescence detection approaches, as listed in Table 2. As seen, most fluorescence sensors for $\mathrm{BHb}$ based on molecular imprinting have been designed with only one fluorescence emission wavelength ${ }^{6,16-18,34,35}$ and the detection limits are slightly higher compared with ratiometric fluorescence systems. Besides, by ratiometric fluorescence technique, visual detection and analysis of $\mathrm{BHb}$ can be achieved. ${ }^{36}$ However, the synthesis process of core-shell composite MIPs ratiometric fluorescence sensor is tedious and time-consuming, since the amount of two different fluorescent materials needs to be optimized. Interestingly, our presented strategy avoided complicated synthesis process, just needed to mix the as-prepared MIPs and $\mathrm{CdTe} @ \mathrm{SiO}_{2}$ within $10 \mathrm{~min}$ for forming dual-emission fluorescence sensor.

\section{CONCLUSIONS}

In summary, we have successfully developed a novel MIR sensor by using CdTe QDs with two different fluorescence colors for the detection of $\mathrm{BHb}$. The green $\mathrm{CdTe} \mathrm{QDs}$ embedded in MIPs was used as responsive signal while the red QDs wrapped in $\mathrm{SiO}_{2}$ was used as reference signal. The sensor relied on the mixture of the two kinds of QDs in a certain proportion. In the presence of $\mathrm{BHb}$, the green MIPs could bind with $\mathrm{BHb}$, leading to fluorescence quenching whereas the red QDs fluorescence remained constant. Thus, the sensor exhibited high selectivity and reliability of visual detection and provided a practical solution for selective recognition and

Table 2. Comparison of Analytical Performances with Other Reported MIPs Fluorescence Sensors for BHb Detection

\begin{tabular}{|c|c|c|c|c|}
\hline system & fluorescence mode & linear range & detection limit & ref \\
\hline UCNPs/MOFs/MIP & single & $0.1-0.6 \mathrm{mg} / \mathrm{mL}$ & $0.062 \mathrm{mg} / \mathrm{mL}$ & 34 \\
\hline Mn-doped ZnS QDs/MIP & single & $0.1-5 \mu \mathrm{M}$ & $0.038 \mu \mathrm{M}$ & 16 \\
\hline Mn-doped ZnS QDs/MIP & single & $0.1-10 \mu \mathrm{M}$ & $0.032 \mu \mathrm{M}$ & 17 \\
\hline $\mathrm{CdTe} /$ thermosensitive MIPs & single & $0.28-4 \mu \mathrm{M}$ & $0.16 \mu \mathrm{M}$ & 18 \\
\hline $\mathrm{Si}-\mathrm{NP} / \mathrm{CdTe} / \mathrm{MIP}$ & single & $0.02-2.1 \mu \mathrm{M}$ & $9.4 \mathrm{nM}$ & 6 \\
\hline $\mathrm{BHb} \mathrm{CdTe} / \mathrm{MIPs}$ & single & $0.3-5.0 \mu \mathrm{M}$ & $0.069 \mu \mathrm{M}$ & 35 \\
\hline $\mathrm{CdTe} @ \mathrm{SiO}_{2} / \mathrm{CdTe} / \mathrm{MIPs}$ ratiometric fluorescence sensor & dual (ratiometric) & $0.02-2.0 \mu \mathrm{M}$ & $6.3 \mathrm{nM}$ & 36 \\
\hline CdTe@MIPs +CdTe@SiO 2 ratiometric fluorescence sensor & dual (ratiometric) & $0.05-3 \mu \mathrm{M}$ & $9.6 \mathrm{nM}$ & this work \\
\hline
\end{tabular}


detection of target protein in biological samples. By virtue of the reasonable mixing of different fluorescence emission materials and fully utilizing the technical superiority of molecular imprinting and ratiometric fluorescence, such type of simple, convenient, and universal strategy would promote the development of MIR sensors for wide applications in separation science, proteomics, and chemo/biosensing.

\section{ASSOCIATED CONTENT}

\section{(S Supporting Information}

The Supporting Information is available free of charge on the ACS Publications website at DOI: 10.1021/acssensors.7b00804.

Fluorescence emission spectra of CdTe@MIPs (blue) and absorption spectra of $\mathrm{BHb}$ (red); statistic particle size distribution of $\mathrm{SiO}_{2}, \mathrm{CdTe} @ \mathrm{MIPs}$, and $\mathrm{CdTe} @$ NIPs; FT-IR of $\mathrm{SiO}_{2}$, CdTe@MIPs, and CdTe@NIP; ratiometric fluorescence response of the MIR sensor upon addition of different amounts of $\mathrm{BHb}$; ratios of CdTe@MIPs and $\mathrm{CdTe} @ \mathrm{SiO}_{2}$; stability of the fluorescence intensity of MIR sensor in $2 \mathrm{~h}$; effect of $\mathrm{pH}$ value on quenching efficiency of MIR sensor in the presence of $\mathrm{BHb}$ at concentration of $1 \mu \mathrm{M}$; fluorescence response time of MIR sensor for $\mathrm{BHb}$ at concentration of $1 \mu \mathrm{M}$; fluorescence intensity recorded at the wavelength of $541 \mathrm{~nm}$; atomic composition ratios (\%) of $\mathrm{SiO}_{2}$, CdTe@MIPs, and CdTe@NIPs from elemental analysis (PDF)

\section{AUTHOR INFORMATION}

\section{Corresponding Authors}

*E-mail: yanxw025@163.com.

*E-mail: lxchen@yic.ac.cn.

\section{ORCID}

Lingxin Chen: 0000-0002-3764-3515

\section{Notes}

The authors declare no competing financial interest.

\section{ACKNOWLEDGMENTS}

This work was financially supported by the National Natural Science Foundation of China (21477160, 21575159, 81573393), the Science and Technology Development Plan of Shandong Province of China (2014GGF01074), the Youth Innovation Promotion Association CAS (2017256), the Defense Science and Technology Innovation Fund of the Chinese Academy of Sciences (CXJJ-17-Q148), and the Instrument Developing Project of the Chinese Academy of Sciences.

\section{REFERENCES}

(1) Duan, L.; He, Q.; Yan, X. H.; Cui, Y.; Wang, K. W.; Li, J. B. Hemoglobin Protein Hollow Shells Fabricated Through Covalent Layer-by-Layer Technique. Biochem. Biophys. Res. Commun. 2007, 354, 357-362.

(2) Duan, H. M.; Wang, X. J.; Wang, Y. H.; Li, J. B.; Luo, C. N. Bioreceptor Multi-walled Carbon Nanotubes@ $\mathrm{Fe}_{3} \mathrm{O}_{4} @ \mathrm{SiO}_{2}-$ Surface Molecular Imprinted Polymer in an Ultrasensitive Chemiluminescent Biosensor for Bovine Hemoglobin. RSC Adv. 2015, 5, 88492-88499.

(3) Wang, Y. Q.; Zhang, H. M.; Zhou, Q. H. Studies on the Interaction of Caffeine with Bovine Hemoglobin. Eur. J. Med. Chem. 2009, 44, 2100-2105.
(4) Chen, L. X.; Wang, X. Y.; Lu, W. H.; Wu, X. Q.; Li, J. H. Molecular Imprinting: Perspectives and Applications. Chem. Soc. Rev. 2016, 45, 2137-2211.

(5) Saylan, Y.; Yilmaz, F.; Özgür, E.; Derazshamshir, A.; Yavuz, H.; Denizli, A. Molecular Imprinting of Macromolecules for Sensor Applications. Sensors 2017, 17, 898.

(6) Li, D. Y.; He, X. W.; Chen, Y.; Li, W. Y.; Zhang, Y. K. Novel Hybrid Structure Silica/CdTe/Molecularly Imprinted Polymer: Synthesis, Specific Recognition, and Quantitative Fluorescence Detection of Bovine Hemoglobin. ACS Appl. Mater. Interfaces 2013, 5, 1260912616.

(7) Zhang, Z.; Li, J.; Wang, X.; Shen, D.; Chen, L. Quantum Dots Based Mesoporous Structured Imprinting Microspheres for the Sensitive Fluorescent Detection of Phycocyanin. ACS Appl. Mater. Interfaces 2015, 7, 9118-9127.

(8) Li, W.; Sun, Y.; Yang, C.; Yan, X.; Guo, H.; Fu, G. Fabrication of Surface Protein Imprinted Nanoparticles Using a Metal Chelating Monomer via Aqueous Precipitation Polymerization. ACS Appl. Mater. Interfaces 2015, 7, 27188-27196.

(9) Li, L.; Lu, Y.; Bie, Z. J.; Chen, H. Y.; Liu, Z. Photolithographic Boronate Affinity Molecular Imprinting: a General and Facile Approach for Glycoprotein Imprinting. Angew. Chem., Int. Ed. 2013, $52,7451-7454$

(10) Wang, S.; Ye, J.; Bie, Z.; Liu, Z. Affinity-tunable Specific Recognition of Glycoproteins via Boronate Affinity-based Controllable Oriented Surface Imprinting. Chem. Sci. 2014, 5, 1135-1140.

(11) Yang, Y. Q.; He, X. W.; Wang, Y. Z.; Li, W. Y.; Zhang, Y. K. Epitope Imprinted Polymer Coating CdTe Quantum Dots for Specific Recognition and Direct Fluorescent Quantification of the Target Protein Bovine Serum Albumin. Biosens. Bioelectron. 2014, 54, 266272.

(12) Li, D. Y.; Zhang, X. M.; Yan, Y. J.; He, X. W.; Li, W. Y.; Zhang, Y. K. Thermo-sensitive Imprinted Polymer Embedded Carbon Dots Using Epitope Approach. Biosens. Bioelectron. 2016, 79, 187-192.

(13) Zhang, K.; Yu, T.; Liu, F.; Sun, M. T.; Yu, H.; Liu, B. H.; Zhang, Z. P.; Jiang, H.; Wang, S. H. Selective Fluorescence Turn-on and Ratiometric Detection of Organophosphate Using Dual-emitting MnDoped ZnS Nanocrystal Probe. Anal. Chem. 2014, 86, 11727-11733.

(14) Qiu, C. X.; Xing, Y. H.; Yang, W. M.; Zhou, Z. P.; Wang, Y. C.; Liu, H.; Xu, W. Z. Surface Molecular Imprinting on Hybrid $\mathrm{SiO}_{2}-$ coated CdTe Nanocrystals for Selective Optosensing of Bisphenol A and its Optimal Design. Appl. Surf. Sci. 2015, 345, 405-417.

(15) Yu, J. L.; Wang, X. Y.; Kang, Q.; Li, J. H.; Shen, D. Z.; Chen, L. $X$. One-pot Synthesis of a Quantum Dot-based Molecular Imprinting Nanosensor for Highly Selective and Sensitive Fluorescence Detection of 4-nitrophenol in Environmental Waters. Environ. Sci.: Nano 2017, 4, 493-502.

(16) Tan, L.; Kang, C.; Xu, S.; Tang, Y. Selective Room Temperature Phosphorescence Sensing of Target Protein Using Mn-doped ZnS QDs-embedded Molecularly Imprinted Polymer. Biosens. Bioelectron. 2013, 48, 216-223.

(17) Tan, L.; Huang, C.; Peng, R.; Tang, Y.; Li, W. Development of Hybrid Organic-inorganic Surface Imprinted Mn-doped ZnS QDs and Their Application as a Sensing Material for Target Proteins. Biosens. Bioelectron. 2014, 61, 506-511.

(18) Zhang, W.; He, X. W.; Li, W. Y.; Zhang, Y. K. Thermo-sensitive Imprinted Polymer Coating CdTe Quantum Dots for Target Protein Specific Recognition. Chem. Commun. 2012, 48, 1757-1759.

(19) Zhang, X.; Yang, S.; Sun, L.; Luo, A. Surface-imprinted Polymer Coating L-cysteine-capped ZnS Quantum Dots for Target Protein Specific Recognition. J. Mater. Sci. 2016, 51, 6075-6085.

(20) Zhang, W.; He, X. W.; Chen, Y.; Li, W. Y.; Zhang, Y. K. Molecularly Imprinted Polymer Anchored on the Surface of Denatured Bovine Serum Albumin Modified CdTe Quantum Dots as Fluorescent Artificial Receptor for Recognition of Target Protein. Biosens. Bioelectron. 2012, 31, 84-89.

(21) Zhang, W.; He, X. W.; Chen, Y.; Li, W. Y.; Zhang, Y. K. Composite of CdTe Quantum Dots and Molecularly Imprinted 
Polymer as a Sensing Material for Cytochrome c. Biosens. Bioelectron. 2011, 26, 2553-2558.

(22) Karfa, P.; Roy, E.; Patra, S.; Kumar, D.; Madhuri, R.; Sharma, P. K. A Fluorescent Molecularly Imprinted Polymer Gate With Temperature and $\mathrm{pH}$ as Inputs for Detection of alpha-fetoprotein. Biosens. Bioelectron. 2016, 78, 454-463.

(23) Tan, L.; Chen, K.; Huang, C.; Peng, R.; Luo, X.; Yang, R.; Cheng, Y.; Tang, Y. A Fluorescent Turn-on Detection Scheme for $\alpha$ fetoprotein Using Quantum Dots Placed in a Boronate-modified Molecularly Imprinted Polymer with High Affinity for Glycoproteins. Microchim. Acta 2015, 182, 2615-2622.

(24) Yao, J. L.; Zhang, K.; Zhu, H. J.; Ma, F.; Sun, M. T.; Yu, H.; Sun, J.; Wang, S. H. Efficient Ratiometric Fluorescence Probe Based on Dual-emission Quantum Dots Hybrid for on-site Determination of Copper Ions. Anal. Chem. 2013, 85, 6461-6468.

(25) Lan, M. H.; Zhang, J. F.; Chui, Y. S.; Wang, P. F.; Chen, X. F.; Lee, C. S.; Kwong, H. L.; Zhang, W. J. Carbon Nanoparticle-based Ratiometric Fluorescent Sensor for Detecting Mercury Ions in Aqueous Media and Living Cells. ACS Appl. Mater. Interfaces 2014, 6, 21270-21278.

(26) Ma, F.; Sun, M. T.; Zhang, K.; Wang, S. H. A Ratiometric Fluorescence Sensor for Highly Selective and Sensitive Detection of Mercuric Ion. Sens. Actuators, B 2015, 209, 377-383.

(27) Xu, S. F.; Lu, H. Z. Ratiometric Fluorescence and Mesoporous Structure Dual Signal Amplification for Sensitive and Selective Detection of TNT Based on MIP@QD Fluorescence Sensors. Chem. Commun. 2015, 51, 3200-3203.

(28) Xu, S. F.; Lu, H. Z. One-pot Synthesis of Mesoporous Sstructured Ratiometric Fluorescence Molecularly Imprinted Sensor for Highly Sensitive Detection of Melamine From Milk Samples. Biosens. Bioelectron. 2015, 73, 160-166.

(29) Lu, H. Z.; Xu, S. F. Visualizing BPA by Molecularly Imprinted Ratiometric Fluorescence Sensor Based on Dual Emission Nanoparticles. Biosens. Bioelectron. 2017, 92, 147-153.

(30) Wang, X. Y.; Yu, J. L.; Kang, Q.; Shen, D. Z.; Li, J. H.; Chen, L. X. Molecular Imprinting Ratiometric Fluorescence Sensor for Highly Selective and Sensitive Detection of Phycocyanin. Biosens. Bioelectron. 2016, 77, 624-630.

(31) Wang, X. Y.; Yu, J. L.; Wu, X. Q.; Fu, J. Q.; Kang, Q.; Shen, D. Z.; Li, J. H.; Chen, L. X. A Molecular Imprinting-based Turn-on Ratiometric Fluorescence Sensor for Highly Selective and Sensitive Ddetection of 2,4-dichlorophenoxyaceticacid(2,4-D). Biosens. Bioelectron. 2016, 81, 438-444.

(32) Yu, Y. L.; Xu, L. R.; Chen, J.; Gao, H. Y.; Wang, S.; Fang, J.; Xu, S. K. Hydrothermal Synthesis of GSH-TGA co-capped CdTe Quantum Dots and Their Application in Labeling Colorectal Cancer Cells. Colloids Surf., B 2012, 95, 247-253.

(33) Turner, N. W.; Piletska, E. V.; Karim, K.; Whitcombe, M.; Malecha, M.; Magan, N.; Baggiani, C.; Piletsky, S. A. Effect of the solvent on recognition properties of molecularly imprinted polymer specific for ochratoxin A. Biosens. Bioelectron. 2004, 20, 1060-1067.

(34) Guo, T.; Deng, Q.; Fang, G.; Gu, D.; Yang, Y.; Wang, S. Upconversion Fluorescence Metal-organic Frameworks Thermosensitive Imprinted Polymer for Enrichment and Sensing Protein. Biosens. Bioelectron. 2016, 79, 341-346.

(35) Wang, X. Y.; Yu, J. L.; Li, J. H.; Kang, Q.; Shen, D. Z.; Chen, L. $X$. Quantum Dots Based Imprinting Fluorescent Nanosensor for the Selective and Sensitive Detection of Phycocyanin: A General Imprinting Strategy toward Proteins. Sens. Actuators, B 2018, 255, 268-274.

(36) Lu, H. Z.; Xu, S. F. Functional Monomer-template-QDs Sandwich Structure for Mesoporous Structured Bovine Hemoglobin Imprinted Ratiometric Fluorescence Sensor. Talanta 2017, 165, 482488 . 